\title{
Vertex-recorded, rather than primary somatosensory cortex-recorded, somatosensory-evoked potentials signal unpleasantness of noxious stimuli in the rat
}

\author{
Peter J. Stienen ${ }^{\mathrm{a}, \mathrm{d}, *}$, Hugo van Oostrom ${ }^{\mathrm{a}, \mathrm{d}}$, Ruud van den Bos ${ }^{\mathrm{c}, \mathrm{d}}$, \\ Harry N.M. de Groot ${ }^{\mathrm{a}}$, Ludo J. Hellebrekers ${ }^{\mathrm{a}}$,b,d \\ a Department of Clinical Sciences of Companion Animals, Section Anaesthesiology and Neurophysiology, Faculty of Veterinary Medicine, \\ Utrecht University, P.O. Box 80.154, NL-3508 TD Utrecht, The Netherlands \\ ${ }^{\mathrm{b}}$ Department of Equine Sciences, Section Anaesthesiology, Faculty of Veterinary Medicine, Utrecht University, \\ P.O. Box 80.153, NL-3508 TD Utrecht, The Netherlands \\ ${ }^{\mathrm{c}}$ Department of Animal, Science and Society, Section Ethology and Welfare, Faculty of Veterinary Medicine, \\ Utrecht University, P.O. Box 80.166, NL-3508 TD Utrecht, The Netherlands \\ ${ }^{\mathrm{d}}$ Rudolf Magnus Institute of Neuroscience, Utrecht University, Utrecht, The Netherlands \\ Received 27 January 2006; received in revised form 18 May 2006; accepted 1 June 2006 \\ Available online 23 June 2006
}

\begin{abstract}
In the present study, we investigated in the rat whether vertex- or primary somatosensory cortex-recorded somatosensory-evoked potentials (VxSEP/SI-SEP, respectively) signal unpleasantness of noxious stimuli. Therefore, initially we characterised fentanyl effects $(0,20,40 \mathrm{or} 50 \mu \mathrm{g} / \mathrm{kg} / \mathrm{h})$ on somatosensory and auditory processing by recording Vx-/SI-SEPs and vertex- and primary auditory cortex-recorded auditory-evoked potentials (Vx-/AI-AEPs, respectively). Subsequently, in a separate experiment, the animals were subjected to a Pavlovian fear-conditioning paradigm. The noxious stimuli applied to evoke Vx-/SI-SEPs (unconditioned stimulus (US)) were paired to a tone (conditioned stimulus (CS)) under 'steady state' conditions of $0,20,40$ or $50 \mu \mathrm{g} / \mathrm{kg} / \mathrm{h}$ fentanyl. Vx-/SI-SEPs were recorded simultaneously during these trials. After CS-US presentation, CS-induced fear-conditioned behaviour was analysed in relation to the SEPs recorded during CS-US presentation and the AEPs recorded in the first experiment. While the SI-SEP and AI-AEP were minimally but significantly affected, fentanyl dose-dependently decreased the Vx-SEP and Vx-AEP. The decrease of the Vx-SEP and Vx-AEP was parallelled by the dose-dependent decrease of the amount of CS-induced fear-conditioned behaviour. These results suggest that the dose-dependent decrease of the Vx-SEP amplitude, rather than of the SI-SEP, indicates that the US was experienced as less unpleasant. Next to an altered US processing, altered CS processing contributed to the decrease of the amount of CS-induced fear-conditioned behaviour as indicated by the dose-dependent decrease of the Vx-AEP.
\end{abstract}

(C) 2006 Elsevier Inc. All rights reserved.

Keywords: SEP; Nociceptive system; Pain; Analgesia; Auditory-evoked potential; Fentanyl

\section{Introduction}

Electroencephalographic somatosensory-evoked potentials (SEPs) evoked by stimulation of peripheral somatosensory fibres appear as a waveform consisting of positive and negative peaks with different amplitudes and time of onset after stimulation (latency). SEP waveforms evoked by noxious stimulation are

\footnotetext{
* Corresponding author. Tel.: +31 30253 1683; fax: +31 302518126

E-mail address: P.Stienen@ vet.uu.nl (P.J. Stienen).
}

considered to represent the processing of the noxious stimulus in the brain $[5,15]$. SEP amplitudes and latencies are altered by drugs primarily suppressing the perception of noxious stimuli (analgesic drugs) $[1,2,15,38]$. Further, in man, SEP amplitudes correlate well with subjective pain ratings $[1,4,14,15,25]$. Combined, the SEP is considered a potential readout-parameter in pain and anaesthesiologic research.

Recently, differences were established between the vertexrecorded positive-to-negative component at approximately 15 and $20 \mathrm{~ms}$ (this component is abbreviated as Vx-SEP throughout this paper) and the primary somatosensory cortex (SI)-recorded 
positive-to-negative component at approximately 12 and $20 \mathrm{~ms}$ (this component is abbreviated as SI-SEP throughout this paper) in the rat model. These differences are of special interest to (anti)nociception and to pain and the absence thereof, i.e. analgesia $[36,38]$. The Vx-SEP and SI-SEP were both found to be related to nociception $[36,38]$. However, the Vx-SEP compared to the SI-SEP, showed an increased sensitivity to filtering out external information and focus attention on newer, more salient stimuli [36,38] In addition, the Vx-SEP showed an increased sensitivity to different 'sleep-inducing' (hypnotic) and analgesic drugs, when compared to the SI-SEP [36,38]. These findings have led to the hypothesis that the Vx-SEP and SI-SEP are related to separate functional pain mechanisms $[36,38]$. While noxious stimuli are evaluated on their spatiotemporal localisation and intensity, their 'emotional color' resulting from signalling unpleasantness, is modulated by the individual's state of arousal, attention and memory [5,21,27,30]. The Vx-SEP is considered to be related to signalling unpleasantness, while the SI-SEP is related to spatio-temporal localisation and intensity quantification of noxious stimuli $[36,38]$.

In the present study, we investigated whether the Vx-SEP, rather than the SI-SEP, signals unpleasantness of noxious stim- uli. Therefore, fentanyl-induced Vx-SEP and SI-SEP changes were studied using the SEP fear-conditioning model [40]. In that previously developed model, stimuli applied to the basal part of the tail to evoke Vx-/SI-SEPs (SEP stimulation paradigm) provided an effective unconditioned stimulus (US) in a fearconditioning paradigm with a tone as conditioned stimulus (CS). It was proposed that the use of a similar approach under different levels of analgesia, would allow to reliably 'translate' analgesic drug-induced SEP changes to its corresponding level of unpleasantness of noxious stimuli (Fig. 1). SEPs represent neural processing of the SEP stimulation paradigm, i.e. the US. Thus analgesic drug-induced SEP changes can be considered to represent changes in US processing during CS-US pairing in our model. The magnitude of the CS-induced fear-response after CS-US pairing, i.e. the strength of the CS-US association represented by the duration of freezing behaviour, is generally accepted as an expression of fear and even considered as an expression of pain experienced from the US [9,32]. Combined, analgesic drug-induced SEP changes during CS-US pairing correlating with a reduced CS-induced fear response, may therefore be assumed to indicate that the US was experienced as less unpleasant by analgesic drug treatment during CS-US pairing (Fig. 1).

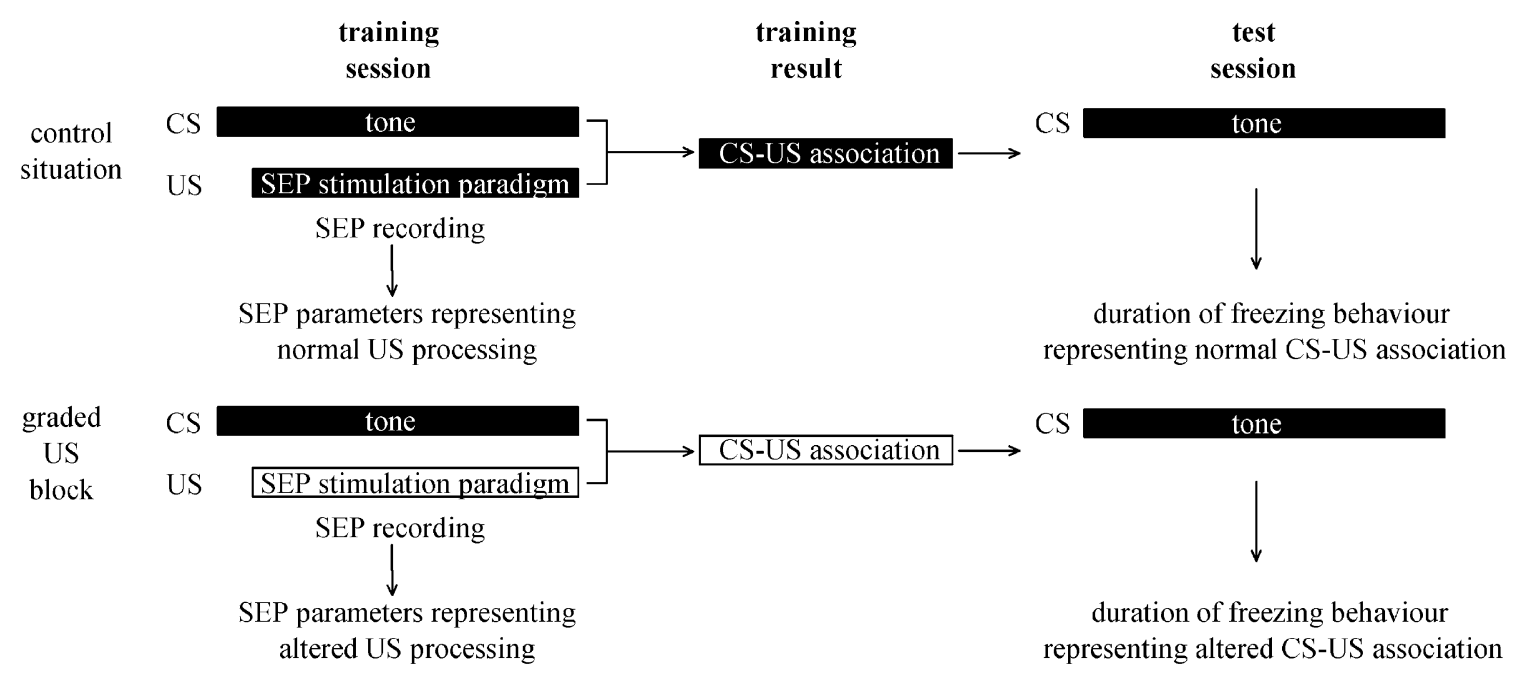

SEP parameters recorded during graded US blocks in training session

vs. corresponding duration of freezing behaviour during test session

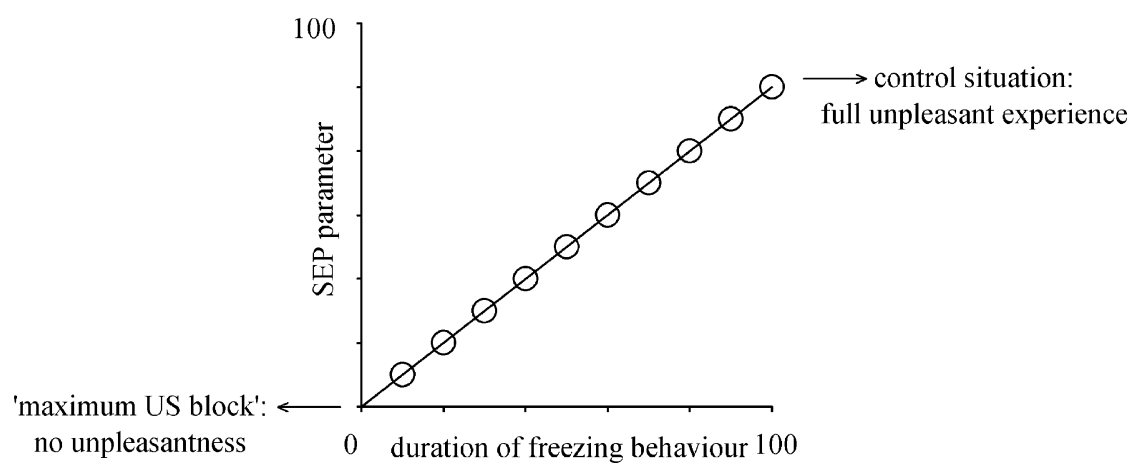

Fig. 1. SEP fear-conditioning model. Upper panel: black and white boxes indicate 'presence of' and 'graded suppression/absence of', respectively. Lower panel: data are arbitrarily chosen based on an 'ideal' situation. For explanation, see text. 
Although fentanyl, a $\mu$-opioid, is primarily analgesic and therefore considered to primarily suppress the perception of unpleasantness of the US, fentanyl may also modulate other sensory processes such as hearing. Consequently, using a tone as CS, fentanyl-induced changes in fear-conditioned behaviour could theoretically be ascribed to effects of fentanyl on neural processing of the CS (CS processing). In order to determine potential fentanyl effects on CS processing, next to neural processing of the US (US processing) and to perform fear-conditioning under 'steady state' conditions of fentanyl, initially we studied the effects of saline and fentanyl $(20,40$ and $50 \mu \mathrm{g} / \mathrm{kg} / \mathrm{h})$ on the Vx-/SI-SEPs and auditoryevoked potentials (AEPs) (Experiment 1) to characterise fentanyl effects on somatosensory and auditory processing and to determine whether a steady state effect on the SEP and AEP parameters could be achieved. In Experiment 2, Vx-/SISEPs were recorded simultaneously during CS-US pairing, according to the SEP-fear conditioning model (Fig. 1), under steady state conditions of saline, 20,40 and $50 \mu \mathrm{g} / \mathrm{kg} / \mathrm{h}$ fentanyl administration. The CS-induced fear-response was tested the day after CS-US pairing and studied in relation to the SEPs and AEPs according to the SEP-fear conditioning model (Fig. 1).

\section{Material and methods}

\subsection{Animals and surgery}

Animal care and experimentation were performed in accordance with protocols approved by the Science Committee and the local Animal Experimentation Committee (Utrecht University, Utrecht, The Netherlands). Adult male Wistar rats (HsdCpb:WU, Harlan Netherlands BV, Zeist, body weight $300-350 \mathrm{~g}, n=62$ ) were pre-medicated with $0.05 \mathrm{mg} / \mathrm{kg}$ atropine sulphate (s.c., Atropine Sulphate $^{\circledR}$, Eurovet Animal Health BV, Bladel, The Netherlands) and, subsequently, anaesthetized with $0.3 \mathrm{mg} / \mathrm{kg}$ fentanyl (i.p., Fentanyl-B. Braun, B. Braun, Melsungen, Germany) and $0.3 \mathrm{mg} / \mathrm{kg}$ medetomidine (i.p., Domitor $^{\circledR}$, Pfizer Animal Health BV, Capelle a/d IJssel, The Netherlands) and fixed in a stereotaxic apparatus (Model 963, Ultra Precise Small Animal Stereotaxic, David Kopf Instruments, Tujunga, CA, USA). In all animals used in Experiments $1(n=9)$ and $2(n=53)$, epidural electrodes (wired stainless steel screws, tip diameter $0.6 \mathrm{~mm}$, impedance $300-350 \Omega$, Fabory DIN 84A-A2, Borstlap BV, Tilburg, The Netherlands) were implanted at the vertex $(4.5 \mathrm{~mm}$ caudal to bregma, $1.0 \mathrm{~mm}$ right from midline [38]), the SI tail representative area $(2.5 \mathrm{~mm}$ caudal to bregma, 2.5 right from midline [7]) and bilateral at the frontal sinus $(10.0 \mathrm{~mm}$ rostral to bregma, $1.0 \mathrm{~mm}$ left and right from midline, respectively [37]). In the animals used in Experiment 1 , an epidural electrode was also inserted at the right primary auditory cortex (AI) $(4.5 \mathrm{~mm}$ caudal to bregma, $4.5 \mathrm{~mm}$ ventral with respect to the dorsal surface of the skull, through the lateral skull [24]). All electrodes were wired to an eight-pin receptacle (Mecap Preci-Dip 917-93-108-41-005, Preci-Dip Durtal SA, Delémont, Switzerland) and fixed to the skull with dental cement (Simplex Rapid, Associated Dental Products, Ltd., Swindon, UK). At the end of surgery, anaesthesia was antagonized with $1 \mathrm{mg} / \mathrm{kg}$ atipamezole (s.c., Antisedan ${ }^{\circledR}$, Pfizer Animal Health BV, Capelle a/d IJssel, The Netherlands) and $0.15 \mathrm{mg} / \mathrm{kg}$ buprenorphine (s.c., Temgesic ${ }^{\circledR}$, ScheringPlough, Amstelveen, The Netherlands). Postoperative analgesia was provided by $0.15 \mathrm{mg} / \mathrm{kg}$ buprenorphine, administered s.c. at $8 \mathrm{~h}$ intervals for 3 days after surgery.

After surgery the animals were housed individually in clear plastic cages under climate-controlled conditions on a reversed 12-h light/12-h dark cycle (lights on at 22:00 h), with ad lib access to food and tap water. Animals were allowed to recover for at least 2 weeks prior to the start of the experiments.

\subsection{Experiment 1: characterisation of fentanyl effects on somatosensory and auditory processing by SEPs and AEPS, respectively}

The experiment was performed in the 'lights off' period in a darkened experimental room. Before the start of the experiment, a 24 gauge cannula (Vasofix ${ }^{\circledR}$, B. Braun, Melsungen, Germany) was inserted in a lateral tail vein of the rat for intravenous administration of saline (injection fluid containing $0.9 \% \mathrm{NaCl}$, Fresenius Kabi B.V., 's Hertogenbosch, The Netherlands) or fentanyl. Next, the rat was fitted in a tight-fitting jacket (developed in house) and individually placed in a purpose-built Plexiglas box with an stainless steel electrically grounded bottom $(40 \mathrm{~cm} \times 28 \mathrm{~cm} \times 30 \mathrm{~cm})$ shielded by a Faraday cage.

For recording of SEPs and AEPs, the rat's head-mounted receptacle was connected, via a swivel connector (SLC-2, Plastics One, Roanoke, VA, USA), to the recording device. SEPs were evoked as previously described in detail [38]. In short, an electrical stimulation device was fixed at the left tail base and wired with a separate electrical shielded cable via the open middle of the swivel connector to the stimulation device. This recording/stimulation set-up prevented electrical interference between recording and electrical stimulation and allowed the animal to move freely inside the box. Stimuli for SEP recording were 72 square-wave pulses of $2 \mathrm{~ms}$ duration with stimulus frequency $0.5 \mathrm{~Hz}$ and stimulus intensity $5 \mathrm{~mA}$ (SEP stimulation paradigm). Stimuli for an AEP recording were 72 white noise clicks of $0.2 \mathrm{~ms}$ duration with stimulus frequency $0.5 \mathrm{~Hz}$ and stimulus intensity $100 \mathrm{~dB}$ sound pressure level and were presented by two speakers mounted in the covering lid of the box.

SEPs were recorded from the vertex and SI (Vx-SEP and SI-SEP, respectively). AEPs were recorded from the vertex and AI (Vx-AEP and AI-AEP, respectively). The accompanying ipsilateral frontal sinus electrode served as reference electrode and the accompanying contralateral frontal sinus electrode served as signal ground. Signals were fed via the swivel connector to separate, but identical, amplifiers (Bio-electric amplifier AB 601-G, Nihon Kohden, Tokyo, Japan). The signals were band-pass filtered between 15 and $300 \mathrm{~Hz}$, amplified 2000 times and fed to a personal computer which digitised the signal online at $2.0 \mathrm{kHz}$. The SEPs and AEPs were at times sensitive to electrical net $50 \mathrm{~Hz}$-interference. Therefore, a $50 \mathrm{~Hz}$ notch-filter was applied to all recording configurations to prevent possible electrical $50 \mathrm{~Hz}$-interference. Power spectral analysis showed that there was a negligible contribution of the $50 \mathrm{~Hz}$-component in regular signals. One SEP/AEP recording consisted of 72 averaged subsequent epochs of 256 data points (pre-stimulus 50 data points, post-stimulus 206 data points) and was stored on disk and analysed offline.

After recording of eight SEPs and AEPs (baseline measurements) over $40 \mathrm{~min}$, the cannula was connected, via tubing, to an automatic anaesthesia pump (Graseby 3500, Graseby Medical Ltd., Watford Herts, UK) and all animals received a continuous infusion over $108 \mathrm{~min}$ of either saline (injection fluid containing $0.9 \% \mathrm{NaCl}$, Fresenius Kabi B.V., 's Hertogenbosch, The Netherlands) or different doses of fentanyl, i.e. 20,40 and $50 \mu \mathrm{g} / \mathrm{kg} / \mathrm{h}$. The infusion time and doses of fentanyl were based upon our own laboratory experience [11,12,38] and pilot experiments preceding this study. Fentanyl was diluted in saline, in a way that all animals received an equal volume of injection fluid during each session. Body temperature was maintained at $37-38^{\circ} \mathrm{C}$ by a water-heating pad placed under the experimental box. The sessions continued until $1 \mathrm{~h}$ after the end of infusion. Recovery time between the sessions was at least 2 weeks and the sequence in receiving either saline or the different dosages of fentanyl, was randomly divided over the animals.

During sessions, every $3 \mathrm{~min}$, the stimulations $(n=72)$ to elicit a SEP or AEP started alternately.

\subsection{Experiment 2: neurophysiologic parameters in relation fear-conditioned behaviour}

Fear-conditioning sessions were performed in the same box and under similar conditions as described in Experiment 1. The box was shielded by polystyrene plates preventing the animals from getting visual cues from outside the fearconditioning box, only leaving access to a camera for observation of the animal's behaviour. The CS (a $40 \mathrm{~s}, 1500 \mathrm{~Hz}$ tone, $85 \mathrm{~dB}$ sound pressure level) was generated by a sound generator (Arbitrary Waveform Generator, Model 33120 A, Hewlett Packard, Palo Alto, CA, USA) and was presented by two speakers 
mounted in the covering lid of the box. The SEP stimulation paradigm (72 square-wave pulses of $2 \mathrm{~ms}$ duration with stimulus frequency $0.5 \mathrm{~Hz}$ and stimulus intensity $5 \mathrm{~mA}$ as in Experiment 1 ) served as US.

Approximately $30 \mathrm{~min}$ prior to the start of the experiment, the animals were placed in the experimental room to acclimatize. Next, a 24 gauge cannula was inserted in a lateral tail vein of the rats for intravenous administration of saline or fentanyl. Subsequently, the rats were fitted in a tight-fitting jacket allowing free movement, and the electrical stimulation device was fixed at the left tail base. Then, the animals were placed in the fear-conditioning box and connected to the stimulation and recording device. Next, the cannula was connected to the automatic anaesthesia pump and infusion of either saline or different doses of fentanyl started. The animals $(n=53)$ were randomly assigned to the following groups: (1) CS-US $0(n=9)$; (2) CS-US $20(n=10)$; (3) CS-US $40(n=8)$; (4) CS-US $50(n=8)$; (5) US $0(n=9)$ and (6) US $50(n=9)$. The CS-US 0 and US 0 group received a continuous infusion of saline. The CS-US 20 and CS-US 40 group received a continuous infusion of 20 and $40 \mu \mathrm{g} / \mathrm{kg} / \mathrm{h}$ fentanyl, respectively. The CS-US 50 and US 50 group both received a continuous infusion of $50 \mu \mathrm{g} / \mathrm{kg} / \mathrm{h}$ fentanyl. Fentanyl was diluted in saline, in a way that all animals in each group received an equal volume of injection fluid. Body temperature was maintained at $37-38^{\circ} \mathrm{C}$ by a water-heating pad placed under the experimental box. Infusion continued in all groups until the end of the training session.

In all groups, fear-conditioning started after $80 \mathrm{~min}$ from the beginning of the infusion according to the results of Experiment 1 (see Section 3.2). The CS-US 0, CS-US 20, CS-US 40 and CS-US 50 groups were each subjected to a Pavlovian fear-conditioning paradigm in which the CS was presented paired with the US. The US started $10 \mathrm{~s}$ after the CS. The interval between the US onsets was $454 \mathrm{~s}$. In the US 0 and US 50 group, the US was presented without the CS using the same interval between US onsets ( $454 \mathrm{~s}$ ). The total number of CS-US pairings per group was 10. In all groups, SEPs were recorded for every US presentation during the fear-conditioning session.

The test sessions were performed in the same experimental room as the fearconditioning sessions under similar conditions and started approximately $24 \mathrm{~h}$ after the end of the training sessions. The sessions were performed, in a Plexiglas box $(41 \mathrm{~cm} \times 31 \mathrm{~cm} \times 20 \mathrm{~cm})$ shielded by polystyrene plates, only leaving access to a camera recording the animal's behaviour. The box was different from the fear-conditioning box in dimensions, and cues at the bottom plate and internal walls. Two speakers in the covering lid of the box presented the CS.

All test sessions were performed during the 'lights off' period. Acclimatization in the experimental room before the actual start of the sessions was $30 \mathrm{~min}$ and in the test apparatus $15 \mathrm{~min}$. During the test sessions, the same CS as used in the training sessions was presented to all groups. The interval between the CS onsets varied between 180 and $480 \mathrm{~s}$. The total number of test trials was 10 .

\subsection{Data and statistical analysis}

Calculations were performed with Microsoft Excel 2000. Statistical analysis was performed with Sigmastat 2.0 and SPSS 11.0. Differences were considered to be significant when $P<0.05$.

\subsubsection{Experiment 1}

SEP and AEP data analysis were performed by visual determination of the prominent components in the SEP and AEP waves of the individual animals. Parameters extracted for analysis were the amplitude of the positive-to-negative components of the (1) Vx-SEP at approximately 15 and $20 \mathrm{~ms}$; (2) SI-SEP at approximately 12 and $20 \mathrm{~ms}$; (3) Vx-AEP at approximately 13 and $20 \mathrm{~ms}$ and (4) AI-AEP at approximately 7 and $10 \mathrm{~ms}$ (see Figs. 1A and 2A, upper trace: Vx-SEP P15-N19, SI-SEP P13-N18, Vx-AEP P14-N19 and AI-AEP P7-N10). Further, a signal-to-noise ratio was calculated as previously described [37]. Peak amplitudes with a signal-to-noise ratio $<2$, were assumed to be zero and were, therefore, replaced by the average noise value in the analysis.

For analysis of drug-effects within and between recording sites, the SEP and AEP amplitudes were expressed as the percentage change with respect to the average baseline measurements ( 8 baseline measurements for both SEP and AEP) for each time point after infusion, each treatment, each recording and each rat separately (relative amplitude). For statistical analysis of drug effect within and between recording sites, the relative amplitude of the SEP and AEP parameters was analysed separately in a three-way repeated analysis of variance
(RM-ANOVA) design including repeated factors treatment (saline, 20, 40 and $50 \mu \mathrm{g} / \mathrm{kg} / \mathrm{h}$ fentanyl), recording (SEP: vertex and SI; AEP: vertex and AI) and time followed by post hoc analysis whenever appropriate.

\subsubsection{Experiment 2}

In Experiment 2, determination of the prominent peaks in the SEP waves and parameters extracted for analysis, were similar as described above. In one animal in the US 50 group, neurophysiologic signals could not be recorded most probably due to a short circuit between the epidural electrodes. This animal was excluded from SEP data analysis. The absolute amplitude $(\mu \mathrm{V})$ of the $\mathrm{Vx}$ SEP P15 and SI-SEP P13, were analysed separately in a two-way RM-ANOVA design including fixed factor group (CS-US 0, CS-US 20, CS-US 40, CS-US 50 , US 0 and US 50) and repeated factor trial followed by post hoc analysis whenever appropriate. For the analysis of drug effects between the recording sites, the SEP data were expressed as relative amplitude (\%). Therefore, the SEP data of the CS-US $0(n=9)$ and US $0(n=9)$ and the SEP data of the CS-US $50(n=8)$ and US $50(n=8)$ were pooled, since there were no significant differences between these groups, respectively (see Section 3.3.1). Then, the data were averaged over the ten trials per rat, since there were no significant trial effects (see Section 3.3.1). Subsequently, the SEP data were expressed as the percentage change with respect to the average of the measurements of the saline group for each recording site and rat, separately. For statistical analysis of the drug effects between the recording sites, the relative amplitude was analysed in a two-way RM-ANOVA design including fixed factor treatment (saline, 20, 40 and $50 \mu \mathrm{g} / \mathrm{kg} / \mathrm{h}$ fentanyl) and repeated factor recording site (vertex and SI) followed by post hoc analysis whenever appropriate.

To study the CS-induced fear-conditioned behaviour in the groups, the amount of freezing behaviour during the presentation of the CS in the test session was evaluated after the experiments using the video-registrations. Freezing behaviour was defined as: the rat adopts a tensed posture with no visible movements except for breathing movements and pendulum motion of the head. Pendulum motion has been described under circumstances of emotional excitement, most often fear [17]. During behavioural evaluation, the experimenter (P.J.S.) was blind with respect to the groups. The behavioural evaluation of the experimenter correlated highly with the evaluation of a second external observer, who was not aware of the aims and procedures of this experiment (Pearson's correlation, $r=0.89, P<0.001, n=35$ ). For statistical analysis, the amount of freezing behaviour was analysed in a two-way RM-ANOVA design including fixed factor group (CS-US 0, CS-US 20, CS-US 40, CS-US 50, US 0 and US 50) and repeated factor trial followed by post hoc analysis whenever appropriate.

\section{Results}

\subsection{General waveforms of the SEPs and AEPs}

Fig. 2A (upper trace) and Fig. 3A (upper trace), show the general average waveforms of the SEPs and AEPs, respectively. Consistent and similar waveforms were recorded across subjects. The traces show a clear Vx-SEP P15 $( \pm 0.19)$ and N19 $( \pm 0.14)$ (rounded to the nearest mean value \pm standard error of the mean (S.E.M)), SI-SEP P13 $( \pm 0.05)$ and N18 $( \pm 0.06)$, VxAEP P14 $( \pm 0.10)$ and N19 $( \pm 0.09)$ and AI-AEP P7 $( \pm 0.05)$ and N10 ( \pm 0.09$)$.

\subsection{Experiment 1: characterisation of fentanyl effects on somatosensory and auditory processing by SEPs and AEPs, respectively}

\subsubsection{Fentanyl effect on SEPS}

The relative amplitudes of the Vx-SEP and SI-SEP (Fig. 2B) were not affected by saline infusion. Both the relative amplitudes of the Vx-SEP and SI-SEP were affected by fentanyl 

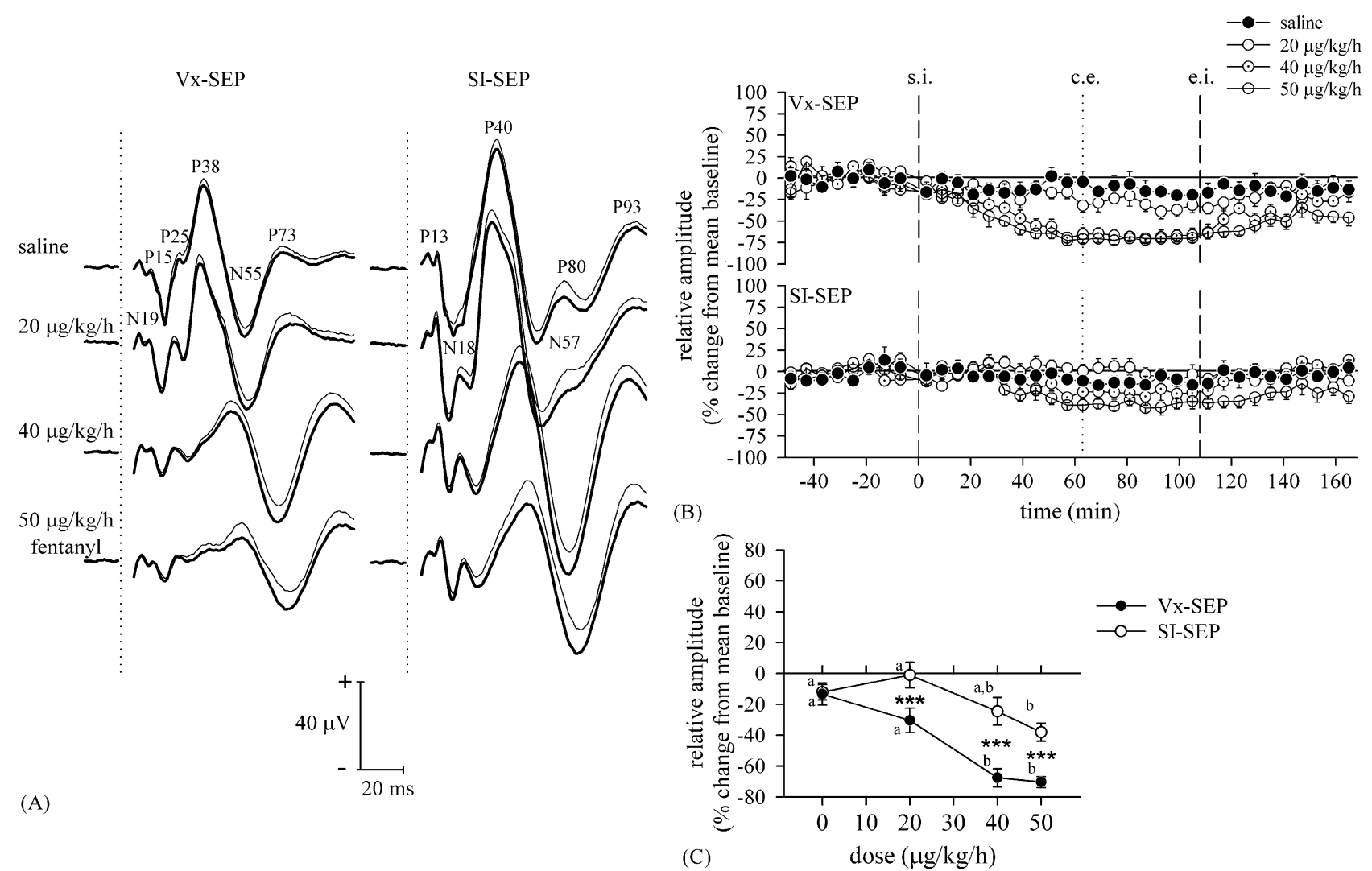

Fig. 2. SEP monitoring during continuous infusion of different doses of fentanyl. (A) Grand mean (bold lines) + S.E.M. (plain lines) waveforms ( $n=9$ ) of the Vx-SEP and SI-SEP during different doses of fentanyl. Dotted line and curve interruption denote stimulus onset. Peaks are designated by either P (positive peak) and N (negative) peak accompanied by their rounded off mean latency (ms). For each animal, the Vx-SEP and SI-SEP resulted from the average of their corresponding SEP recordings during the period in which continuous infusion of fentanyl resulted in a constant effect on the SEP parameters. (B) Effect of fentanyl on the SEP parameters. Data are represented as the mean relative amplitude $(\% ; \pm$ S.E.M.; $n=9)$ over time following infusion of either saline or 20,40, or $50 \mu \mathrm{g} / \mathrm{kg} / \mathrm{h}$. s.i. $=\mathrm{start}$ infusion; c.e. = time point that fentanyl infusion resulted in a constant effect on the SEP parameters; e.i. = end infusion. (C) Differences between the effect of fentanyl on the Vx-SEP and SI-SEP in the period after fentanyl infusion resulted in a constant effect on the SEP parameters. Data are represented as the mean relative amplitude ( \pm S.E.M., $n=9$ ) against the dose of fentanyl. Data points corresponding to either Vx-SEP or SI-SEP with the same characters are not different from one another (one-way ANOVA, factor dose followed by SNK). ${ }^{* * *} P<0.001$ (Vx-SEP vs. SI-SEP; post hoc paired $t$-test).

treatment over time, but their latencies were negligibly affected (Fig. 2A and B). Visual observation showed both the Vx-SEP and SI-SEP to be stable at $63 \mathrm{~min}$ after infusion onset of the different treatments. Following the end of the different fentanyl infusions, both parameters gradually recovered over time (Fig. 2B).

Overall statistical analysis over the period from $63 \mathrm{~min}$ to the end of infusion $(t=108 \mathrm{~min})$ showed that the relative amplitude of the Vx-SEP and SI-SEP during all treatments, were not affected over time (treatment $\times$ recording $\times$ time: $F(21,168)=0.748, P=0.778$; treatment $\times$ time: $F(21,168)=$ $0.694, P=0.835$; recording $\times$ time: $F(7,56)=0.648, P=0.714$; time: $F(7,56)=1.033, P=0.419)$, but were significantly and differently affected by the specific treatments (treatment $\times$ recording: $F(3,24)=11.355, P<0.001)$. Post hoc analysis showed that the relative amplitude of the Vx-SEP was significantly lower during 40 and $50 \mu \mathrm{g} / \mathrm{kg} / \mathrm{h}$ fentanyl compared to saline and $20 \mu \mathrm{g} / \mathrm{kg} / \mathrm{h}$ fentanyl (Fig. 2C) (one-way RM-ANOVA, factor treatment: $F(3,24)=19.558, P<0.001$ followed by Student-Newman-Keuls for post hoc comparison (SNK), $P<0.05)$. The relative amplitude of the SI-SEP was significantly lower during $50 \mu \mathrm{g} / \mathrm{kg} / \mathrm{h}$ compared to saline and $20 \mu \mathrm{g} / \mathrm{kg} / \mathrm{h}$ fentanyl (Fig. 2C) (one-way RM-ANOVA, factor treatment: $F(3,24)=4.952, P=0.008$ followed by SNK, $P<$ $0.05)$.

The relative amplitude of the Vx-SEP during 20, 40 and $50 \mu \mathrm{g} / \mathrm{kg} / \mathrm{h}$ fentanyl was significantly lower compared to the relative amplitude of the SI-SEP (Fig. 2C) (paired $t$-test: $20 \mu \mathrm{g} / \mathrm{kg} / \mathrm{h}$ fentanyl: $t(8)=-5.093, P=0.001 ; 40 \mu \mathrm{g} / \mathrm{kg} / \mathrm{h}$ fentanyl: $t(8)=-4.988, P=0.001$ and $50 \mu \mathrm{g} / \mathrm{kg} / \mathrm{h}: t(8)=-9.543$, $P<0.001)$.

\subsubsection{Fentanyl effect on AEPs}

The relative amplitudes of the Vx-AEP and AI-AEP were not affected by saline infusion (Fig. 3B). The relative amplitude of the Vx-AEP was affected by fentanyl treatment, whereas the latency of the Vx-AEP and the AI-AEP and the relative amplitude of the AI-AEP were negligibly affected (Fig. 3A and B). Visual observation showed both parameters to be stable at 69 min after infusion onset of the different treatments. After the end of infusion, both parameters gradually recovered over time (Fig. 3B). 


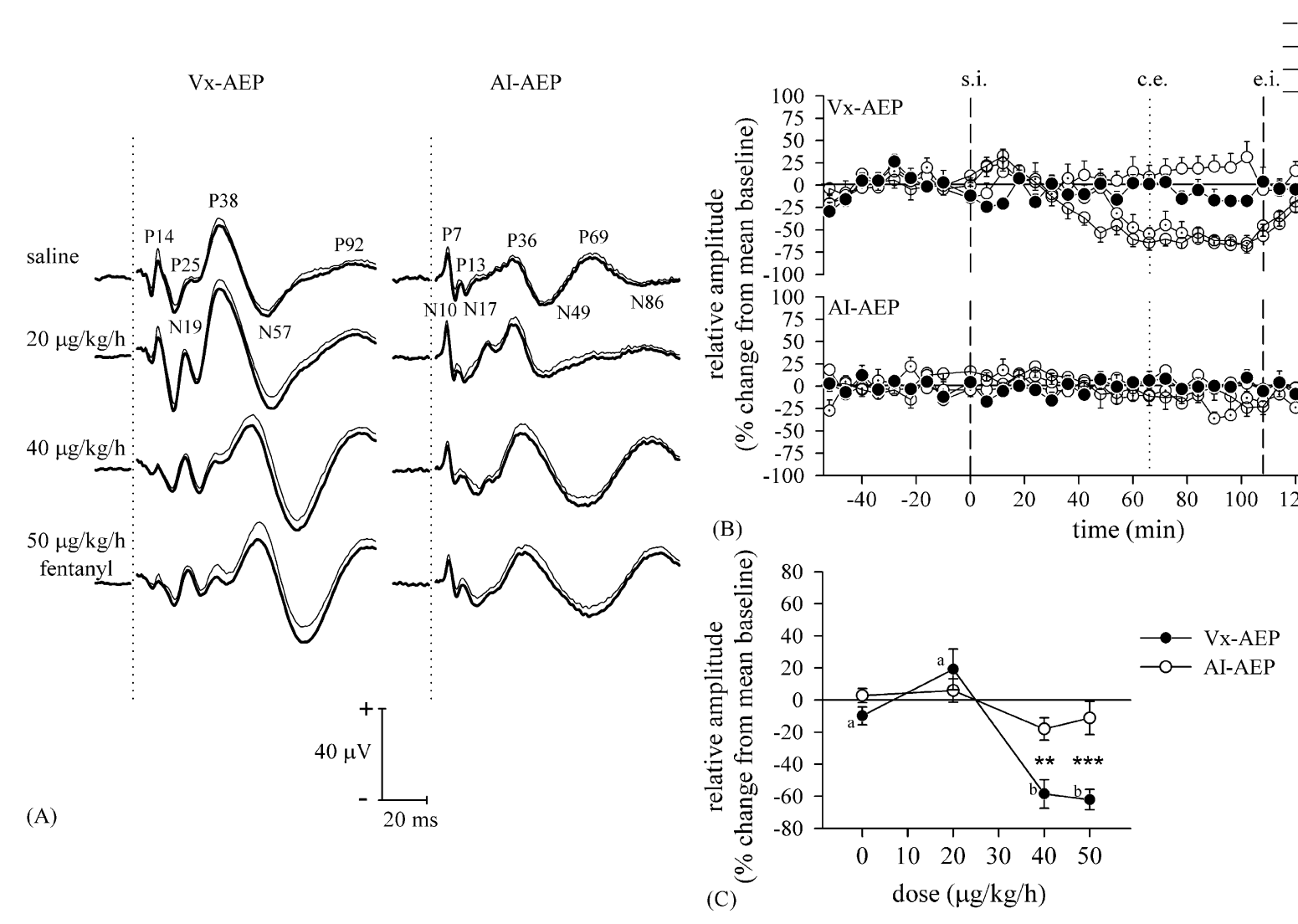

Fig. 3. AEP monitoring during continuous infusion of different doses of fentanyl. (A) Grand mean (bold lines) + S.E.M. (plain lines) waveforms $(n=9)$ of the Vx-AEP and AI-AEP during different doses of fentanyl. Dotted line and curve interruption denote stimulus onset. Peaks are designated by either P (positive peak) and N (negative) peak accompanied by their rounded off mean latency (ms). For each animal, the Vx-AEP and AI-AEP resulted from the average of their corresponding SEP recordings during the period in which continuous infusion of fentanyl resulted in a constant effect on the AEP parameters. (B) Effect of fentanyl on the AEP parameters. Data are represented as the mean relative amplitude $(\% ; \pm$ S.E.M.; $n=9)$ over time following infusion of either saline or 20,40 , or $50 \mu \mathrm{g} / \mathrm{kg} / \mathrm{h}$. s.i. $=\mathrm{start}$ infusion; c.e. $=$ time point that fentanyl infusion resulted in a constant effect on the AEP parameters; e.i. $=$ end infusion. $(\mathrm{C})$ Differences between the effect of fentanyl on the Vx-AEP and AI-AEP. Data are represented as the mean relative amplitude ( \pm S.E.M., $n=9$ ) against the dose of fentanyl. Data points corresponding to either Vx-AEP or AI-AEP with the same characters are not different from one another (one-way ANOVA, factor dose followed by SNK). ${ }^{* *} P<0.01,{ }^{* * *} P<0.001(\mathrm{Vx}-\mathrm{SEP}$ vs. SI-SEP; post hoc paired $t$-test).

Overall statistical analysis over the period from $69 \mathrm{~min}$ to the end of infusion showed that the relative amplitude of the Vx-AEP and AI-AEP during all treatments, were not affected over time (treatment $\times$ recording $\times$ time: $F(18,144)=1.010, \quad P=0.453$; treatment $\times$ time: $\quad F(18,144)=1.267, \quad P=0.218 ; \quad$ recording $\times$ time: $F(6,48)=0.155, P=0.987$; time: $F(6,48)=0.917$, $P=0.491)$, but were significantly and differently affected by the different treatments (treatment $\times$ recording: $F(3,24)=12.179$, $P<0.001)$. Post hoc analysis showed that the relative amplitude of the Vx-AEP was significantly lower during 40 and $50 \mu \mathrm{g} / \mathrm{kg} / \mathrm{h}$ fentanyl compared to saline and $20 \mu \mathrm{g} / \mathrm{kg} / \mathrm{h}$ fentanyl (Fig. 3C) (one-way RM-ANOVA, factor treatment: $F(3,24)=22.816$, $P<0.001$ followed by SNK, $P<0.05)$. No differences existed between the relative amplitude of the AI-AEP during the different treatments (Fig. 3C) (one-way RM-ANOVA, factor treatment: $F(3,24)=2.774, P=0.063)$.

The relative amplitude of the Vx-AEP was significantly lower during 40 and $50 \mu \mathrm{g} / \mathrm{kg} / \mathrm{h}$ fentanyl, when compared to the relative amplitude of the AI-AEP (Fig. 3C) (paired $t$-test: $40 \mu \mathrm{g} / \mathrm{kg} / \mathrm{h}$ fentanyl: $t(8)=-3.581, P=0.007$ and $50 \mu \mathrm{g} / \mathrm{kg} / \mathrm{h}$ : $t(8)=-5.594, P=0.001)$.
3.3. Experiment 2: SEP-fear conditioning model: neurophysiologic parameters in relation fear-conditioned behaviour

\subsubsection{SEP parameters}

During fear-conditioning, overall statistical analysis showed that for all groups, the absolute amplitude of both the $\mathrm{Vx}$-SEP and SI-SEP were not affected over trials (Fig. 4A and B) (Vx-SEP amplitude: trial $\times$ group: $F(45,414)=0.887, P=0.681$; trial: $F(9,45)=1.650, P=0.099$; SI-SEP amplitude: trial $\times$ group: $F(45,414)=1.220, P=0.164$; trial: $F(9,45)=1.342, P=0.213)$. The absolute amplitude of the Vx-SEP was different between the groups, whereas the absolute amplitude of the SI-SEP was not different between the groups (Fig. 4A and B) (Vx-SEP amplitude: group: $F(5,46)=11.514, P<0.001$; SI-SEP amplitude: group: $F(5,46)=2.132, P=0.078)$. Post hoc analysis showed that the amplitude of the Vx-SEP in the CS-US 40, CS-US 50 and US 50 group was significantly lower, when compared to the CS-US 0, CS-US 20 and US 0 group (Fig. 4A) (SNK: $P<$ $0.05)$. 

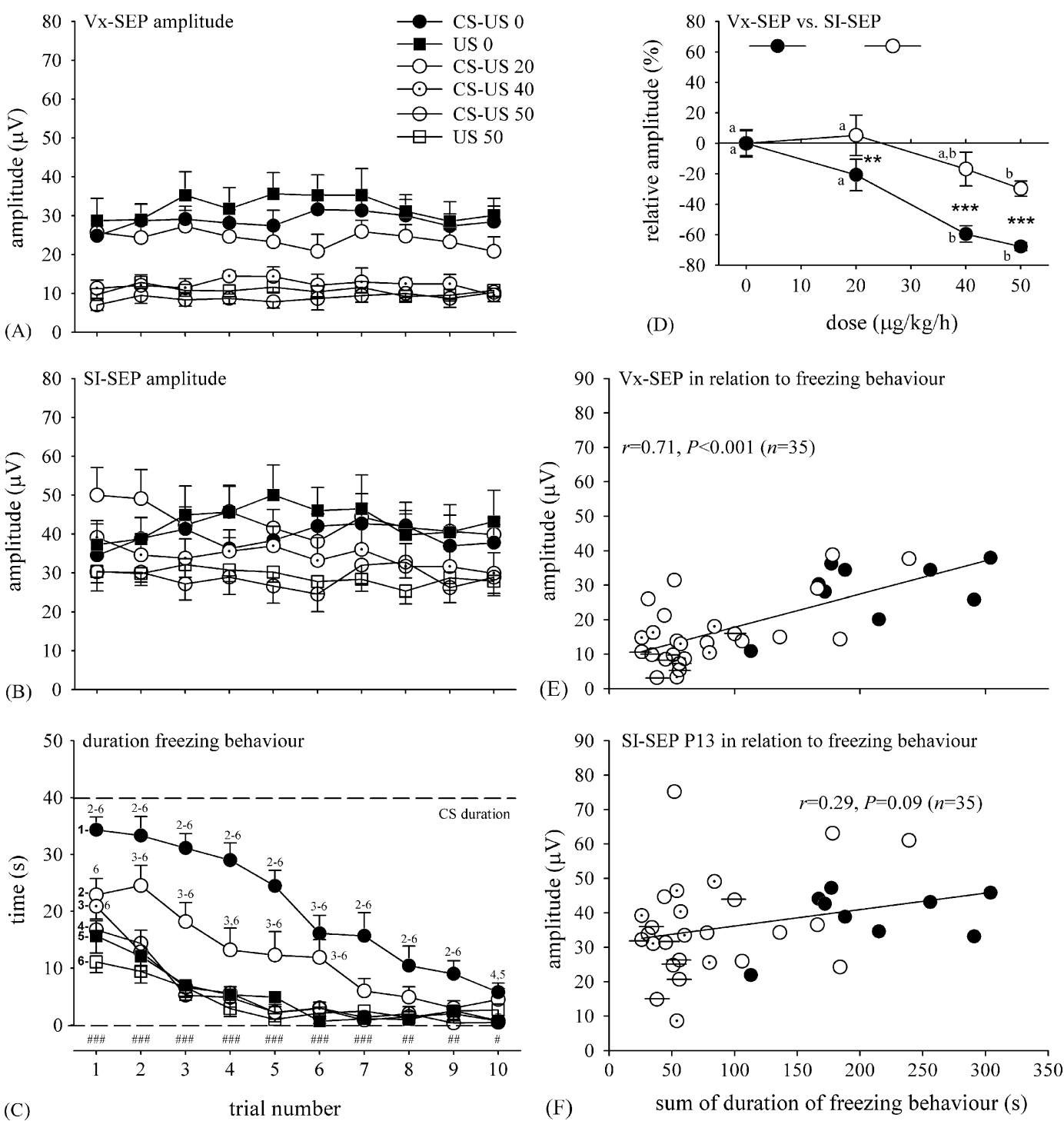

Fig. 4. Effect of fear-conditioning during continuous infusion of different doses of fentanyl on SEP parameters and fear-conditioned behaviour. (A) Effect of fearconditioning on the amplitude of the Vx-SEP in the different treated groups. Data are represented as the mean amplitude $(\mu \mathrm{V} ; \pm$ S.E.M.) over the ten training trials. (B) Effect of fear-conditioning on the amplitude of the SI-SEP in the different treated groups. Data are represented as the mean amplitude ( $\mu$ V; \pm S.E.M.) over the ten training trials. (C) Duration of freezing behaviour in the different groups during CS presentation. Data are represented as the mean duration of freezing behaviour (s; \pm S.E.M.) over the subsequent test trials after fear-conditioning. ${ }^{\#} P<0.05,{ }^{\# \#} P<0.01,{ }^{\# \#} P<0.001$ (one-way ANOVA, factor dose; the numbers (1 to 6) refer to significant differences compared to the group(s) that are designated by that number (post hoc SNK)). (D) Differences between the effect of fentanyl on the Vx-SEP and SI-SEP during fear-conditioning. Data are represented as the mean relative amplitude $(\% ; \pm$ S.E.M.) against the dose of fentanyl $($ dose $(\mu \mathrm{g} / \mathrm{kg} / \mathrm{h}): 0$ (saline): $n=18 ; 20: n=10 ; 40: n=8$ and 50: $n=16$ ). Data points corresponding to either Vx-AEP or ACx-AEP with the same characters are not different from one another (one-way ANOVA, factor dose followed by SNK). ${ }^{* *} P<0.01,{ }^{* * *} P<0.001$ (Vx-SEP vs. SI-SEP; post hoc paired $t$-test). (E) Pearson product-moment correlation of averaged (10 trials) Vx-SEP amplitude $(\mu \mathrm{V})$ and the sum (10 trials) of duration of freezing behaviour (s). (F) Pearson product-moment correlation of averaged (10 trials) SI-SEP amplitude $(\mu \mathrm{V})$ and the sum (10 trials) of duration of freezing behaviour (s).

When expressed as the percentage amplitude change with respect to the mean amplitude of the saline group, overall statistical analysis showed that the relative amplitude of the Vx-SEP and of the SI-SEP were affected differently by the different treatments (Fig. 4D) (treatment $\times$ recording: $F(3,48)=14.773$, $P<0.001)$. Post hoc analysis showed that the relative amplitude of the Vx-SEP was significantly lower during 40 and $50 \mu \mathrm{g} / \mathrm{kg} / \mathrm{h}$ fentanyl compared to saline and $20 \mu \mathrm{g} / \mathrm{kg} / \mathrm{h}$ fentanyl (Fig. 4D) (one-way ANOVA, factor treatment: Vx-SEP: $F(3,48)=19.300$, $P<0.001$ followed by SNK, $P<0.05)$. The relative amplitude of the SI-SEP was significantly lower during $50 \mu \mathrm{g} / \mathrm{kg} / \mathrm{h}$ fentanyl compared to saline and $20 \mu \mathrm{g} / \mathrm{kg} / \mathrm{h}$ fentanyl (one-way ANOVA, factor treatment: SI-SEP: $F(3,48)=3.518, P=0.022)$.

The relative amplitude of the Vx-SEP was significantly lower during 20, 40 and $50 \mu \mathrm{g} / \mathrm{kg} / \mathrm{h}$ fentanyl compared to the SI-SEP P13 (Fig. 4D) (paired $t$-test: $20 \mu \mathrm{g} / \mathrm{kg} / \mathrm{h}$ fentanyl: $t_{9}=-3.340, P=0.009 ; 40 \mu \mathrm{g} / \mathrm{kg} / \mathrm{h}$ fentanyl: $t_{7}=-5.926$, $P<0.001$ and $50 \mu \mathrm{g} / \mathrm{kg} / \mathrm{h}$ fentanyl $\left.t_{15}=-9.945, P<0.001\right)$.

\subsubsection{Freezing behaviour}

Although the tone induced freezing behaviour in all groups, the duration of freezing behaviour differed between the 
groups over trials (Fig. 4C) (trial $\times$ group: $F(45,423)=3.832$, $P<0.001$ ). Post hoc analysis showed that the duration of freezing behaviour in all groups, decreased significantly over the trials (one-way RM ANOVA, factor trial: $P<0.001$ in all groups). In the CS-US 0 group, the duration of freezing behaviour overall was significantly longer over the trials, when compared to the CS-US 20, CS-US 40, CS-US 50, US 0 and US 50 group (Fig. 4C) (one-way ANOVA, factor group followed by SNK: see Fig. $4 \mathrm{C}$ for results). Further, although in the first trial, the duration of freezing behaviour in the CS-US 20 group was similar compared to CS-US 40, CS-US 50 and US 0 group, in trial 2-6, the duration of freezing behaviour in the CS-US 20 group overall was significantly longer and the extinction rate of freezing behaviour was significantly less, when compared to the CS-US 40, CS-US 50, US 0 and US 50 group (Fig. 4C) (one-way ANOVA, factor group followed by SNK: see Fig. 4C for results). The duration of freezing behaviour displayed by the different groups, was highly correlated with the level of the Vx-SEP amplitude (Fig. 4E) (Pearson's correlation, $r=0.71, n=35, P<0.001$ ), whereas correlation between the duration of freezing behaviour and the level of the SI-SEP amplitude was absent (Fig. 4F) (Pearson's correlation, $r=0.29$, $n=35, P=0.09$ ). Finally, the correlation coefficient resulting from the correlation analysis between the duration of freezing behaviour and the Vx-SEP amplitude, was significantly different from that of the correlation analysis between the duration of freezing behaviour and the SI-SEP amplitude (Fisher $r$-to- $z$ transformation, $z=2.35, P=0.019$ ).

\section{Discussion}

The major findings of the present study are, firstly, that fentanyl dose-dependently decreased the amplitude of the VxSEP, while the SI-SEP was minimally, although significantly, affected. These results support that the Vx-SEP and SI-SEP are related to nociception as previously reported $[36,38]$. Both noxious and tactile responses can be found within the $10-30 \mathrm{~ms}$ latency range $[5,13,31,33]$. However, with $\mu$-opiate receptor agonists like fentanyl modulating noxious, rather than tactile, processing $[6,16,34,41,42]$, the Vx-SEP and SI-SEP in the present study can be considered to represent processing of noxious stimuli. Secondly, fentanyl dose-dependently decreased the CS-induced fear-response as measured during the test sessions. This decrease correlated with the dose-dependent decrease of the Vx-SEP, rather than the SI-SEP. Combined, we suggest that the dose-dependent decrease of the Vx-SEP amplitude, rather than of the SI-SEP, indicates that the US was experienced as less unpleasant.

\subsection{Fear-conditioned behaviour and the formation of a CS-US association}

The tone-induced freezing behaviour in the US 0 and US 50 group in the present study, explicitly represents a 'novelty' response and cannot be related to CS-US association, as no CS was presented during training. The duration of freezing behaviour of the CS-US 0 and CS-US 20 group was signifi- cantly greater than the duration of freezing behaviour of the US 0 and US 50 group. Therefore, the duration of freezing behaviour of the CS-US 0 and CS-US 20 group can be ascribed to the formation of a CS-US association during conditioning, rather than being a 'novelty' response.

The duration of freezing behaviour of the CS-US 40 and CS-US 50 group was not different with respect to that of the US 0 and US 50 group, but was different with respect to that of the CS-US 0 and CS-US 20 group. Therefore, we suggest that the CS-US 40 and CS-US 50 group formed no CS-US association during fear-conditioning.

The fact that the tone-induced freezing behaviour of the US 50 group was similar to that of the US 0 group suggests that fentanyl does not induce a relevant effect on auditory processing and behavioural expression during testing afterwards, i.e. does not increase or decrease freezing behaviour due to a difference in the perception of tones. Therefore, the duration of freezing behaviour in the CS-US 20, CS-US 40 and CS-US 50 group, can be ascribed to fentanyl-induced effects on the formation of a CS-US association during fear-conditioning, rather than to fentanyl-induced effects on tone processing and behavioural expression during testing afterwards.

\subsection{Neurophysiologic parameters in relation to fear-conditioned behaviour}

The effects of fentanyl on the Vx-SEP amplitude, rather than on the SI-SEP, correlated with the fentanyl-induced effects on the formation of a CS-US association. Therefore, the fentanylinduced dose-dependent reduction of the Vx-SEP amplitude, rather than of the SI-SEP, is suggested to be related to a reduced perception of the US indicating that the US was experienced as less unpleasant. Analgesic drug-induced SEP changes can be considered to represent analgesic drug-induced changes in US processing, and the magnitude of the CS-induced fearresponse, can be considered a measure of the animal's adversity to the US during fear-conditioning. Combined, analgesic druginduced SEP changes during fear-conditioning, that correlate with a reduced CS-induced fear-response after conditioning, i.e. the changes of the Vx-SEP rather than of the SI-SEP in the present study, may therefore be assumed to indicate that the US was experienced as less unpleasant.

The AEP results in the present study suggest that, next to an effect on US processing, fentanyl affected CS processing during fear-conditioning. This might have contributed to the less distinct or absence of the formation of a CS-US association in the CS-US 20 and CS-US 40 and CS-US 50 group, respectively, during fear-conditioning.

The Vx-AEP can be considered a specific measure of arousaldependent perceptual awareness of auditory stimuli. Considerable evidence exists on the involvement of the pedunculopontine nucleus (PPN) and/or its medial thalamic output projections in the activating reticular system (RAS), in the generation of the Vx-AEP component occuring at $11-15 \mathrm{~ms}$ (P14-N19 in the present study) $[22,23,28,29,39]$. The AI-AEP occuring at 6-9 ms (P7-N10 in the present study), is well established as primary cortical excitation of the AI in the primary auditory 
pathway [3]. PPN to medial thalamic output projections have been implicated in arousal to auditory stimuli (i.e. 'wake -up, something happened') [10,35], whereas the AI is involved in auditory spatio-temporal localisation and intensity quantification (i.e. 'what is it') [8]. Therefore, the decreases in Vx-AEP amplitude rather than in the AI-AEP in Experiment 1 in the present study, can be considered to be related to a reduced perception of auditory stimuli, and thus, indicate fentanyl effects on CS processing during fear-conditioning in Experiment 2. It should be noted here that studying possible fentanyl effects on auditory processing by AEPs during fear-conditioning following the SEP fear-conditioning model [40] was experimentally limited. AEPs are evoked by short, repeated auditory stimuli rather than a continous tone like the CS, which, consequently, is not useful to evoke AEPs during fear-conditioning.

Since following $20 \mu \mathrm{g} / \mathrm{kg} / \mathrm{h}$ fentanyl administration, the $\mathrm{VX}$ AEP amplitude was not affected, the less distinct CS-US association in the CS-US 20 group, is to be ascribed to effects of fentanyl on US processing, rather than CS processing. This is in accordance with the fact that $\mu$-opioids such as fentanyl are primarily analgesic $(20 \mu \mathrm{g} / \mathrm{kg} / \mathrm{h}$ fentanyl in the present study), and therefore, can be considered to primarily suppress processing of nociceptive stimuli (US), rather than other sensory processes (CS). The absence of the CS-US association in the CS-US 40 and CS-US 50 group, can be ascribed to an effect of fentanyl on CS processing, next to US processing, since the Vx-AEP amplitude was decreased by 40 and $50 \mu \mathrm{g} / \mathrm{kg} / \mathrm{h}$ fentanyl. This is in accordance with the fact that $\mu$-opioids at higher doses (40 and $50 \mu \mathrm{g} / \mathrm{kg} / \mathrm{h}$ fentanyl in the present study) do modulate other sensory processes including auditory processing.

From the present data, the possibility that fentanyl affects the ability of associating the CS and US at the level of the amygdala, which is the locus for formation and storage of CS-US associations during fear-conditioning $[18,20]$ cannot be excluded. Therefore, next to the effects of fentanyl on US and CS processing represented by decreases of the Vx-SEP and Vx-AEP amplitude, respectively, the possibility that fentanyl affects associating the CS and US at the level of the amygdala, might potentially have contributed to the less distinct or absence of the formation of a CS-US association in the CS-US 20 and CS-US 40 and CS-US 50 group, respectively, during fear-conditioning.

An alternative, albeit unlikely, influence on the fentanylinduced effects on the CS-US association in the CS-US 20, CS-US 40 and CS-US 50 groups, might be found in statedependent learning. This refers to the situation whereby information learned while the animal is under the influence of a certain drug ('state'), such information can be recalled only when the animal is in the same 'state' [26]. However, this phenomenon is most often very weak or absent $[19,26]$ and therefore, is highly unlikely to have a significant a role in the present study.

\subsection{Conclusions}

In summary, the fact that the dose-dependent decrease of the Vx-SEP amplitude, rather than the SI-SEP, was paralleled by a dose-dependent decrease of the duration of freezing behaviour, suggests that the Vx-SEP, rather than the SI-SEP, signals unpleasantness of noxious stimuli, and thus is potentially a useful and reliable readout-parameter in pain and anaesthesiologic research. For a meaningful interpretation of the SEPs in pain assessment, in future studies, the Vx-/SI-SEP is to be investigated in relation to the structure and function of separate functional nociceptive pathways relaying either unpleasantness or spatio-temporal localisation and intensity quantification of noxious stimuli according to the possible mechanisms underlying the differences between the Vx-SEP and SI-SEP as recently described in full detail $[36,38]$.

\section{References}

[1] L. Arendt-Nielsen, Characteristics, detection, and modulation of laserevoked vertex potentials, Acta Anaesthesiol. Scand. Suppl. 101 (1994) $7-44$.

[2] M. Banoub, J.E. Tetzlaff, A. Schubert, Pharmacologic and physiologic influences affecting sensory evoked potentials: implications for perioperative monitoring, Anesthesiology 99 (2003) 716-737.

[3] D.S. Barth, S. Di, Three-dimensional analysis of auditory-evoked potentials in rat neocortex, J. Neurophysiol. 64 (1990) 1527-1536.

[4] P. Bjerring, L. Arendt-Nielsen, Argon laser induced single cortical responses: a new method to quantify pre-pain and pain perceptions, J. Neurol. Neurosurg. Psychiatry 51 (1988) 43-49.

[5] B. Bromm, J. Lorenz, Neurophysiological evaluation of pain, Electroencephalogr. Clin. Neurophysiol. 107 (1998) 227-253.

[6] K.L. Casey, P. Svensson, T.J. Morrow, J. Raz, C. Jone, S. Minoshima, Selective opiate modulation of nociceptive processing in the human brain, J. Neurophysiol. 84 (2000) 525-533.

[7] J.K. Chapin, C.S. Lin, Mapping the body representation in the SI cortex of anesthetized and awake rats, J. Comp. Neurol. 229 (1984) 199213

[8] F. de Ribaupierre, Acoustical information processing in the auditory thalamus and cerebral cortex, in: G. Ehret, R. Romand (Eds.), The Central Auditory System, Oxford University Press, New York, 1997, pp. 317-397.

[9] M.S. Fanselow, R.C. Bolles, Naloxone and shock-elicited freezing in the rat, J. Comp. Physiol. Psychol. 93 (1979) 736-744.

[10] E. Garcia-Rill, Y. Homma, R.D. Skinner, Arousal mechanisms related to posture and locomotion: 1. Descending modulation, Prog. Brain Res. 143 (2004) 283-290.

[11] Z.L. Haberham, W.E. van den Brom, A.J. Venker-van Haagen, V. Baumans, H.N. de Groot, L.J. Hellebrekers, EEG evaluation of reflex testing as assessment of depth of pentobarbital anaesthesia in the rat, Lab. Anim. 33 (1999) 47-57.

[12] Z.L. Haberham, W.E. van den Brom, A.J. Venker-van Haagen, H.N. de Groot, V. Baumans, L.J. Hellebrekers, The rat vertex-middle latency auditory-evoked potential as indicator of anaesthetic depth: a comparison with evoked-reflex testing, Brain Res. 873 (2000) 287290.

[13] B. Heppelmann, M. Pawlak, S. Just, R.F. Schmidt, Cortical projection of the rat knee joint innervation and its processing in the somatosensory areas SI and SII, Exp. Brain Res. 141 (2001) 501-506.

[14] G.D. Iannetti, M. Leandri, A. Truini, L. Zambreanu, G. Cruccu, I. Tracey, Adelta nociceptor response to laser stimuli: selective effect of stimulus duration on skin temperature, brain potentials and pain perception, Clin. Neurophysiol. 115 (2004) 2629-2637.

[15] R. Kakigi, S. Watanabe, H. Yamasaki, Pain-related somatosensory evoked potentials, J. Clin. Neurophysiol. 17 (2000) 295-308.

[16] J. Kalliomaki, X.L. Luo, Y.B. Yu, J. Schouenborg, Intrathecally applied morphine inhibits nociceptive $\mathrm{C}$ fiber input to the primary somatosensory cortex (SI) of the rat, Pain 77 (1998) 323-329.

[17] V.G. Kolpakov, P.M. Borodin, N.N. Barykina, Catatonic behaviour in the Norway rat, Behaviour 62 (1977) 190-208. 
[18] J. LeDoux, The emotional brain, fear, and the amygdala, Cell Mol. Neurobiol. 23 (2003) 727-738.

[19] J.H. Maes, J.M. Vossen, State-dependency of conditioning and extinction of an appetitive response with amphetamine and midazolam, Pharmacol. Biochem. Behav. 58 (1997) 305-310.

[20] S. Maren, Neurobiology of Pavlovian fear conditioning, Annu. Rev. Neurosci. 24 (2001) 897-931.

[21] M.J. Millan, The induction of pain: an integrative review, Prog. Neurobiol. 57 (1999) 1-164.

[22] H. Miyazato, R.D. Skinner, T. Crews, K. Williams, E. Garcia-Rill, Serotonergic modulation of the P13 midlatency auditory evoked potential in the rat, Brain Res. Bull. 51 (2000) 387-391.

[23] H. Miyazato, R.D. Skinner, E. Garcia-Rill, Neurochemical modulation of the P13 midlatency auditory evoked potential in the rat, Neuroscience 92 (1999) 911-920.

[24] H. Miyazato, R.D. Skinner, N.B. Reese, F.A. Boop, E. Garcia-Rill, A middle-latency auditory-evoked potential in the rat, Brain Res. 37 (1995) 247-255.

[25] S. Ohara, N.E. Crone, N. Weiss, R.D. Treede, F.A. Lenz, Amplitudes of laser evoked potential recorded from primary somatosensory, parasylvian and medial frontal cortex are graded with stimulus intensity, Pain 110 (2004) 318-328.

[26] D. Overton, Contextual stimulus effects of drugs and internal states, in: P.D. Balsam, A. Tomie (Eds.), Context and Learning, Lawrence Erlbaum, Hillsdale, NJ, 1985, pp. 357-384.

[27] D.D. Price, Psychological and neural mechanisms of the affective dimension of pain, Science 288 (2000) 1769-1772.

[28] N.B. Reese, E. Garcia-Rill, R.D. Skinner, Auditory input to the pedunculopontine nucleus: I. Evoked potentials, Brain Res. Bull. 37 (1995) 257-264.

[29] N.B. Reese, E. Garcia-Rill, R.D. Skinner, Auditory input to the pedunculopontine nucleus: II. Unit responses, Brain Res. Bull. 37 (1995) 265-273.

[30] A. Schnitzler, M. Ploner, Neurophysiology and functional neuroanatomy of pain perception, J. Clin. Neurophysiol. 17 (2000) 592-603.

[31] J. Schouenborg, J. Kalliomaki, P. Gustavsson, I. Rosen, Field potentials evoked in rat primary somatosensory cortex (SI) by impulses in cutaneous A beta- and C-fibres, Brain Res. 397 (1986) 86-92.
[32] T.V. Sewards, M.A. Sewards, The medial pain system: neural representations of the motivational aspect of pain, Brain Res. Bull. 59 (2002) 163-180.

[33] F.Z. Shaw, R.F. Chen, H.W. Tsao, C.T. Yen, Comparison of touch- and laser heat-evoked cortical field potentials in conscious rats, Brain Res. 824 (1999) 183-196.

[34] S.C. Silbert, D.W. Beacham, E.W. McCleskey, Quantitative single-cell differences in mu-opioid receptor mRNA distinguish myelinated and unmyelinated nociceptors, J. Neurosci. 23 (2003) 34-42.

[35] R.D. Skinner, Y. Homma, E. Garcia-Rill, Arousal mechanisms related to posture and locomotion: 2. Ascending modulation, Prog. Brain Res. 143 (2004) 291-298.

[36] P.J. Stienen, H.N.M. de Groot, A.J. Venker-van Haagen, W.E. van den Brom, L.J. Hellebrekers, Differences between somatosensory-evoked potentials recorded from the ventral posterolateral thalamic nucleus, primary somatosensory cortex and vertex in the rat, Brain Res. Bull. 67 (2005) 269-280.

[37] P.J. Stienen, Z.L. Haberham, W.E. van den Brom, H.N.M. de Groot, A.J. Venker-Van Haagen, L.J. Hellebrekers, Evaluation of methods for eliciting somatosensory-evoked potentials in the awake, freely moving rat, J. Neurosci. Methods 126 (2003) 79-90.

[38] P.J. Stienen, W.E. van den Brom, H.N.M. de Groot, A.J. Venker-van Haagen, L.J. Hellebrekers, Differences between primary somatosensory cortex- and vertex-derived somatosensory-evoked potentials in the rat, Brain Res. 1030 (2004) 256-266.

[39] L. Teneud, H. Miyazato, R.D. Skinner, E. Garcia-Rill, Cholinergic modulation of the sleep state-dependent P13 midlatency auditory evoked potential in the rat, Brain Res. 884 (2000) 196-200.

[40] H. van Oostrom, P.J. Stienen, R. van den Bos, H.N.M. de Groot, L.J. Hellebrekers, Development of a rat model to assess the efficacy of the somatosensory-evoked potential as indicator of analgesia, Brain Res. Brain Res. Protoc. 15 (2005) 14-20.

[41] D.C. Yeomans, B.Y. Cooper, C.J. Vierck, Effects of systemic morphine on responses of primates to first or second pain sensations, Pain 66 (1996) 253-263.

[42] D.C. Yeomans, V. Pirec, H.K. Proudfit, Nociceptive responses to high and low rates of noxious cutaneous heating are mediated by different nociceptors in the rat: behavioral evidence, Pain 68 (1996) 133-140. 